\title{
Size-filtering effects by stacking InAs/InP (001) self-assembled quantum wires into multilayers
}

\author{
B. Alén and J. Martínez-Pastor* \\ Instituto de Ciencia de los Materiales, Universidad de Valencia, P.O. Box 2085, 46071 Valencia, Spain \\ L. González and J. M. García \\ Instituto de Microelectrónica de Madrid (CNM, CSIC), Isaac Newton 8, 28760 Tres Cantos, Madrid, Spain
}

S. I. Molina, A. Ponce, and R. García

Departamento de Ciencia de los Materiales e I. M. y Q. I., Universidad de Cádiz, 11510 Puerto Real, Cádiz, Spain

(Received 14 September 2001; revised manuscript received 28 December 2001; published 29 May 2002)

\begin{abstract}
Multilayer structure containing vertically stacked InAs/InP self-assembled quantum wires have been successfully grown by molecular-beam epitaxy. The influence of the InP spacer layer thickness on the structural and optical properties of the wire superlattice has been studied by means of transmission electron microscopy and photoluminescence. The coherent propagation of the strain field in the sample with a 5-nm-thick spacer determines by a size filtering effect a good homogeneity and uniformity of the wire stacks, and hence a good optical quality. The exciton recombination dynamics in the wire superlattice cannot be related to thermal escape of carriers out to the barriers, as occurs in single layer samples.
\end{abstract}

DOI: 10.1103/PhysRevB.65.241301

In the last few years a considerable effort has been devoted to the achievement of uniform self-assembled quantum dots and wires, given the technological advantages predicted for these systems in optoelectronic devices. The stacking of quantum dots in multilayer structures has been proposed as an efficient mechanism to improve uniformity. ${ }^{1,2}$ This effect has been demonstrated in the case of InAs/GaAs selfassembled quantum dots, where deposition of multiple layers of InAs separated by GaAs spacers results in vertically correlated quantum dots of better structural and optical properties. $1,3-5$

Spontaneous formation of InAs quantum wires (QWR's) on InP (001) has been recently demonstrated..$^{6-11}$ These nanostructures are potential candidates for applications in light-emission devices working at $\lambda \approx 1.55 \mu \mathrm{m}$. ${ }^{11,12}$ The formation of InAs QWR's, instead of quantum dots, is due to the buildup of a strong stress anisotropy at the InP/InAs interface under molecular-beam epitaxy (MBE) growth conditions. ${ }^{13}$ Multilayer structures based on the InAs/InP system have been also grown by using $\mathrm{In}_{0.52} \mathrm{Al}_{0.48} \mathrm{As}$, $\mathrm{In}_{0.53} \mathrm{Ga}_{0.47} \mathrm{As}$, and $\mathrm{In}_{0.52} \mathrm{Al}_{0.48} \mathrm{As} / \mathrm{In}_{0.53} \mathrm{Ga}_{0.47} \mathrm{As}$ short period superlattices as spacers. ${ }^{6,8,9}$ The wires in these structures are either vertically correlated or anticorrelated in the different layers, depending on the composition of the spacer, probably due to demixing effects of the InAlAs spacer. ${ }^{8}$

Using InP as the spacer material, in this work we present evidences of a strain driven vertical filtering of the wire sizes in stacked InAs self-assembled QWR multilayers, namely, wire superlattice (WSL). The influence of the spacer thickness in the structural and optical properties of the WSL has been studied by means of transmission electron microscopy (TEM) and photoluminescence (PL).

The samples studied here consist of a stack of ten layers containing self-assembled InAs QWR's separated by InP spacer layers with different thickness ( $5 \mathrm{~nm}$ for sample $A$ and $12.5 \mathrm{~nm}$ for sample $B$ ), grown by MBE on $\operatorname{InP}[001]$ substrates. The InAs layers were grown at a substrate temperature $T_{S}=400^{\circ} \mathrm{C}$, whereas the InP buffer and InP spacer lay-
PACS number(s): 73.21.Hb, 78.55.Cr, 78.67.Lt

ers were grown at $T_{S}=450{ }^{\circ} \mathrm{C}$. We used a growth rate of 0.44 monolayers per second (ML/s) and a beam equivalent pressure (BEP) $\left(A s_{4}\right)=3 \times 10^{-6}$ mbar for InAs and BEP $\left(P_{2}\right)=7 \times 10^{-6}$ mbar for InP. After deposition of each 4-ML-thick InAs layer in the structure, the growth was interrupted during $60 \mathrm{~s}$ and $T_{S}$ increased up to $470{ }^{\circ} \mathrm{C}$ for enabling self-assembling process. Similar samples, but containing single QWR layers, both with and without InP capping, have been also studied in previous works and used here for comparison with the WSL's. ${ }^{11-13}$ In these samples, the nominal thickness of the deposited InAs layers was 2.5 ML.

TEM studies were carried out in a Jeol 1200 EX microscope operated at $120 \mathrm{kV}$ and a Jeol $2000 \mathrm{EX}$ working at 200 $\mathrm{kV}$ with structural resolution of $0.21 \mathrm{~nm}$. Cross-sectional TEM (XTEM) specimens were prepared by mechanical thinning and ion milling at $\mathrm{N}_{2}$ liquid temperature to minimize damage of InP. Photoluminescence experiments in the 50$250 \mathrm{~K}$ range were carried out by using the 514-nm line of an $\mathrm{Ar}^{+}$laser. The PL signal was dispersed by a $\frac{1}{8}-\mathrm{m}$ focal length double monochromator and synchronously detected by an extended InGaAs $p-i-n$ photodiode.

Bright field XTEM images of samples $A$ and $B$ recorded under two beams condition exciting the 002 reflection are shown in Fig. 1. XTEM studies were also carried out keeping the electron beam along the $\mathrm{InP}[1 \overline{1} 0]$ direction, that is, along the QWR axis. These images allow the on-axis observation of QWR's, though such image conditions do not maximize the chemical contrast between InAs and InP. To improve such contrast, 002 reflections have to be excited without exciting other less chemically sensitive reflections (e.g., 111 reflections). This is done by tilting the sample to get the electron beam along another crystalline direction such as $[3 \overline{2} 0]$. This direction is contained in the plane [001] and the spots with maximum intensity correspond to $002 n$ and $00 \overline{2} n$ reflections. Such a high-resolution image is shown on the bottom of Fig. 1(a) for a selected area of the stack ensemble, as indicated. We can see how the contrast between 

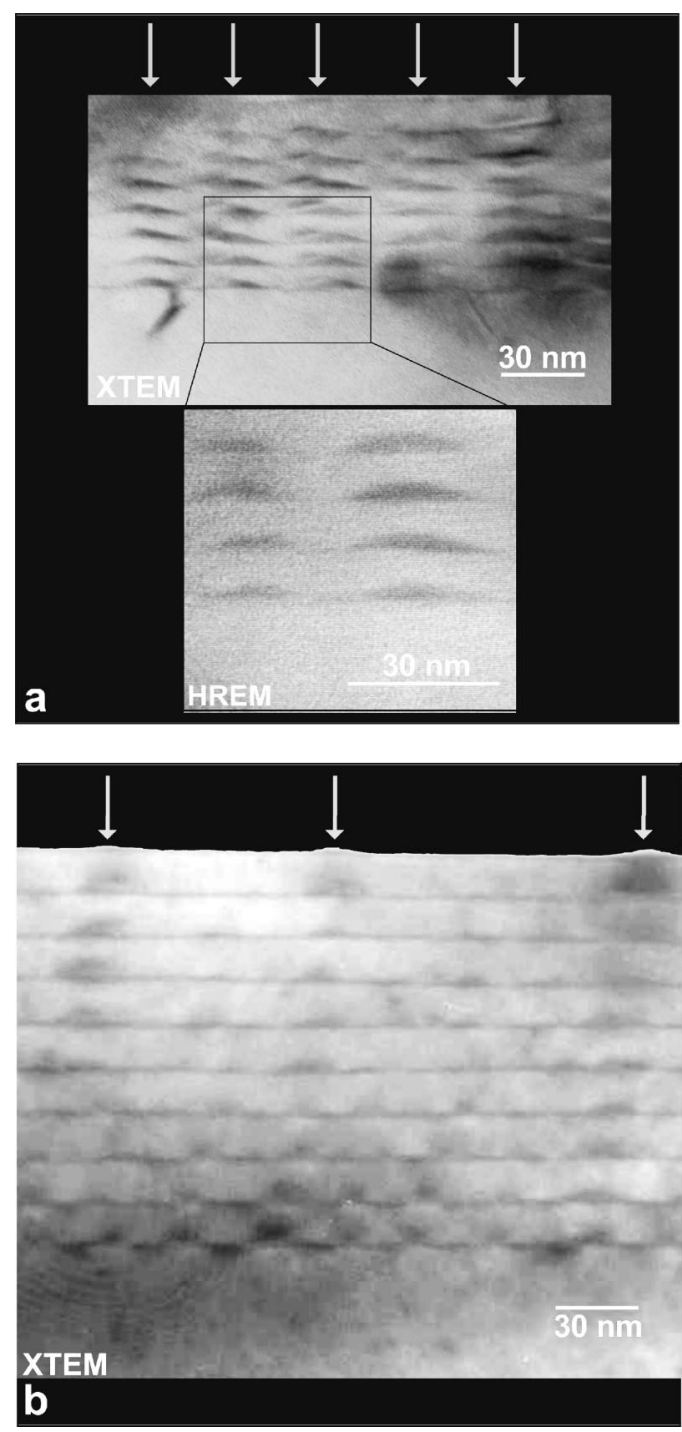

FIG. 1. XTEM images of samples $A$ (a) and $B$ (b). The selected area in the XTEM image of sample $A$ is also shown under highresolution conditions at the bottom side of (a).

the InAs wires and the InP spacers and buffer is really improved, and the wire shape better defined with respect to the XTEM image of the same zone.

Table I lists all the geometric parameters of the wire stack system in samples $A$ and $B$, i.e., height $(H)$ and width $(W)$ of the wires, in-plane spacing $(\lambda)$, and spacer layer thickness $(S)$. These parameters were measured from on-axis [110] and $[3 \overline{2} 0]$ (three beams) XTEM images of an area containing eight stacks of ten wires in both samples. The error given in all these parameters corresponds to the standard deviation (80 data). The average value measured of $(S)$ in the wire intervalleys is in good agreement with nominal values. Also included in Table I are the average values found from atomic force microscopy (AFM) micrographs taken on InAs single layer uncapped samples. The difference in height between uncapped and stacked QWR can be due to the different amount of InAs deposited and also to the error introduced by the AFM estimate of the shape.

From Fig. 1 and Table I, we can establish the following
TABLE I. Height $(H)$, base width $(W)$, period $(\lambda)$, and spacer layer thickness $(S)$ of InAs QWR's measured by XTEM in stacks of 10 layers (samples $A$ and $B$ ) and by AFM in uncapped single layer (single QWR) are shown.

\begin{tabular}{lcccc}
\hline \hline Sample & $(H)(\mathrm{nm})$ & $(W)(\mathrm{nm})$ & $\lambda(\mathrm{nm})$ & $(S)(\mathrm{nm})$ \\
\hline Single QWR $^{\mathrm{a}}$ & $0.6-2$ & $18 \pm 2$ & $24 \pm 2$ & \\
$A^{\mathrm{b}}$ & $3.4 \pm 0.6$ & $16.1 \pm 1.7$ & $27 \pm 3$ & $3.9 \pm 0.5$ \\
$B^{\mathrm{b}}$ & $4.3 \pm 1.2$ & $17 \pm 3$ & $74 \pm 16$ & $11.7 \pm 0.7$ \\
\hline \hline
\end{tabular}

${ }^{\mathrm{a}}$ From AFM images.

${ }^{\mathrm{b}}$ From XTEM images.

findings: (1) In sample $A$ most of the wires in the first layer propagates vertically giving rise to aligned and uniform stacks of wires. The uniformity of the wires in the eight stacks is measured by $\Delta H / H$ and $\Delta W / W$, which are around $18 \%$ and $10 \%$, respectively. It is worth noting that $\Delta H$ is equal to $2 \mathrm{ML}$, in agreement with the linewidth of the main PL line, as discussed below. The uniformity of the spatial distribution of the stacks is measured by $\Delta \lambda / \lambda$, also around a $10 \%$. The absolute value of $\lambda_{A}$ is close to the in-plane pitch period found in single InAs QWR layers. A deviation lower than $2^{\circ}$ is measured between the first and the last wire of the stack. (2) In contrast, in sample $B$, one of the three wires $\left(\lambda_{A} / \lambda_{B} \sim 3\right)$ in the first layer propagates vertically, giving rise to QWR stacks. This nucleating wire is particularly big, namely, a "defect" wire. The uniformity of the wires in the stacks is two times worse than in the sample $A$, as also for the spatial distribution $\left(\Delta \lambda_{B} / \lambda_{B}\right.$ around $\left.22 \%\right)$. The deviation measured from the first to the last wire in the stack is now around $6^{\circ}$, three times greater than in sample $A$.

Therefore, the above given results demonstrates how the 5 -nm-thick spacer is thin enough for enabling an efficient strain propagation along the growth direction through the whole structure. The wires are clearly piled up in a correlated way, as for InAs/GaAs quantum dot multilayers, ${ }^{1,3-5}$ because the spacer layers are made of a binary compound (InP) and demixing effects cannot appear. Our results provide further experimental evidence for the model proposed in Ref. 8 to explain the anticorrelation effects. At the same time, the strain redistribution between wires and spacer layer has a filtering effect on the size of the wires being stacked, giving rise to the observed vertical $\left(H_{A}\right)$ and lateral $\left(W_{A}, \lambda_{A}\right)$ uniformity, in contrast to the observed findings in sample $B$, as outlined above. The larger thickness $(12.5 \mathrm{~nm})$ of the InP spacer layers in sample $B$ inhibits the strain propagation between consecutive layers and the wires in vertical stacks are only $1 / 3$ of the total, promoted by "defect" wires in the first layer. Optical results will be in agreement with the structural characterization, as discussed below.

Figure 2 shows the PL spectra for two single layer QWR samples [Fig. 2(a)], and samples $A$ [Fig. 2(b)] and $B$ [Fig. 2(c)]. All the spectra have been normalized for clarity, but it is interesting to note that the PL peak intensities are comparable in all cases. The PL peak intensity of sample $B$ (no size filtering) is a factor 2 greater than in sample $A$ (size filtering). In the single layer case the PL band can be deconvoluted in several Gaussian components, as shown in Fig. 2(a) for the 


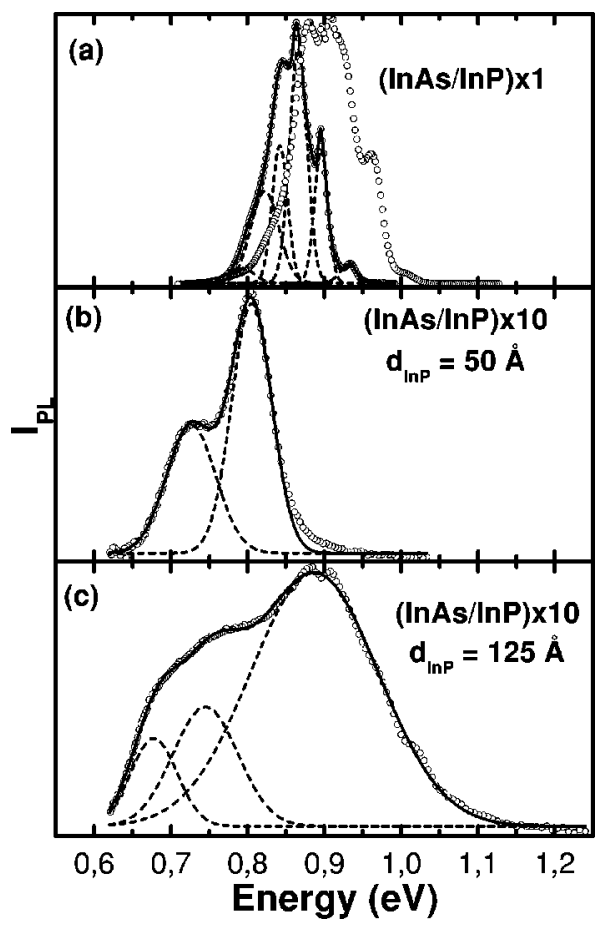

FIG. 2. PL spectra at $50 \mathrm{~K}$ of (a) two samples containing a single QWR layer, (b) sample $A$ and (c) sample $B$.

best quality sample. Both samples have been grown under similar conditions by depositing the same thickness of InAs, nominally $2.5 \mathrm{ML}$, but they do not exhibit the same degree of uniformity. By correlating AFM and approximate calculations of the band to band optical transitions, the Gaussian components of the spectra in Fig. 2(a) can be attributed to different QWR families, each one representative of a wire height. ${ }^{11,12}$ Two consecutive components arise from 1-ML fluctuation in the wire height, and the range of the absolute wire height assignment, within 1-2 ML accuracy, approximately, ${ }^{12}$ is $8-13 \mathrm{ML}$, from high- to low-energy components in the best quality sample, and 7-13 ML in the other one. These samples will be used here for comparison with samples $A$ and $B$, being the most important parameters for such comparison the central emission energy and the linewidth and peak energy of the Gaussian components (representative of a QWR height family).

In sample $A$ we can deconvolute two Gaussian components, centered at 0.728 and $0.805 \mathrm{eV}$, being the latter the dominant one, as shown in Fig. 2(b). The linewidths of such components are around $50 \mathrm{meV}$, approximately the double of the linewidths deduced for single-height family emission lines in the single layer samples. In this way, each emission component in sample $A$ would arise from a stack family in which the wire height fluctuations are around 2 ML $(0.6 \mathrm{~nm})$, in agreement with the standard deviation found in the eight stacks measured by XTEM for this sample (Table I). In contrast, in sample $B$ we find a broad emission band at $0.89 \mathrm{eV}$ and two not well resolved and narrower Gaussian components at 0.678 and $0.745 \mathrm{eV}$, as shown in Fig. 2(c). From XTEM data, we can assign the broad band to the dominant vertically uncorrelated wires, $2 / 3$ of the total. Indeed the central energy of this band is close to that measured in the single layer samples [see Fig. 2(a)]. The larger InAs amount used in sample $B(4 \mathrm{ML})$ and the residual strain field between different layer stacks are the main differences from single layer samples, and they could be the origin of the absence of structure in the $0.89-\mathrm{eV}$ emission band in sample $B$. The lowenergy bands would be thus associated to vertical stacks of wires, in a $1 / 3$ proportion, as given by XTEM measurements. The overall linewidth of these poorly resolved bands, are compatible with the 4-ML (1.2-nm) standard deviation of the wire height found in the wires of the eight examined stacks in sample $B$ (Table I). At the same time, the redshift of these bands is consequent with the larger average wire height found in the stacks of this sample $(4.3 \mathrm{~nm})$ with respect to the average value found in sample $A(3.4 \mathrm{~nm})$, for which we associate the main emission band at $0.805 \mathrm{eV}$ shown in Fig. 2(b). On the one hand, the ground optical transition lies at around $0.807 \mathrm{eV}$ in the case of a QWR $12 \mathrm{ML}(3.6 \mathrm{~nm})$ high. ${ }^{12}$ On the other hand, the energy-level splitting due to vertical coupling of the electron wave functions, and hence the expected redshift of the corresponding emission for that wire size, can be of the order of $20 \mathrm{meV}{ }^{14}$ Therefore, the association of the $0.805-\mathrm{eV}$ band in sample $A$ to stacks in which the wires are $3.4 \mathrm{~nm}$ high (near to $11 \mathrm{ML}$ ), in average, is completely reasonable.

More important than these electronic structure considerations, also sensitive to the accuracy in our model (1-2 ML), is the optical quality improvement found from sample $B$ to sample $A$ due to the size filtering effect of the spacer layer. The broad band centered at $0.89 \mathrm{eV}$ in sample $B$ (vertically uncorrelated wires) is representative of height fluctuations greater than $9 \mathrm{ML}$ in the wire ensemble (more than nine families not resolved), by comparing with the single layer samples. In sample $A$ we find two families of QWR stacks emitting at 0.728 and $0.805 \mathrm{eV}$. The latter is dominant and is assigned to the QWR stacks measured by XTEM. The height fluctuations within these two families of stacks is not greater than $2 \mathrm{ML}$, according to the structural and optical characterization. The size fluctuations are thus reduced by more than a factor 2 in sample $A$ by using a narrower spacer layer.

Figure 3 shows the temperature evolution of the emission in samples $A$ [Fig. 3(a)] and $B$ [Fig. 3(b)]. The different recombination dynamics taking place in vertically correlated and uncorrelated wires can be observed on sample $B$ where both coexist [Fig. 3(b)], as referred above. In our previous work on single QWR layers, thermal escape of carriers towards the barriers, where they recombine nonradiatively, was demonstrated to be the main mechanism affecting the emission efficiency at high temperatures. ${ }^{12}$ In that work we showed that the high-energy PL components (smaller wires) quench faster than the low-energy ones (taller wires), given the smaller energy difference between the gap barrier and the excitonic optical transitions in the first case. The activation energies of the PL quenching were thus different from highto low-energy components, raging from 100 to $350 \mathrm{meV}$. A similar phenomenology is observed in the case of sample $B$, where we observe how the broad Gaussian band at $0.89 \mathrm{eV}$ is quenched comparatively much faster than the low-energy features, as shown in Fig. 3(b). This is a further experimental evidence of the origin of this band, associated to different 


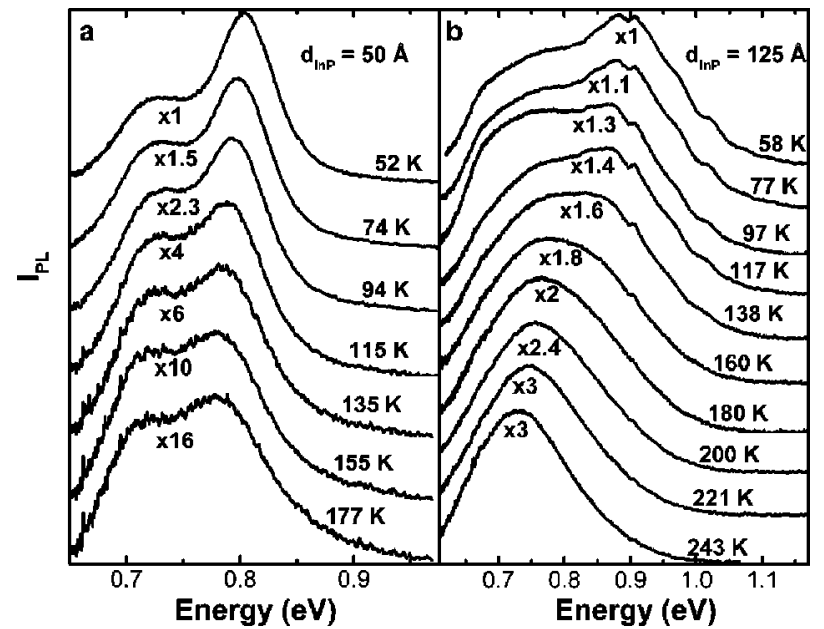

FIG. 3. Temperature evolution of the PL spectra in samples $A$ (a) and $B$ (b).

QWR families not vertically correlated, even if no doubt can arise after the evidences discussed above. The temperature evolution of the PL components associated to vertically correlated wires in sample $B$ cannot be appropriately obtained from data plotted on Fig. 3(b), because they mix between them and with the PL signal coming from vertically uncorrelated wires above $140 \mathrm{~K}$. The exciton recombination dynamics in the WSL can be better observed in sample $A$, where vertically correlated wires are dominant. The highenergy component $(0.805 \mathrm{eV})$ quenches faster than the lowenergy one $(0.728 \mathrm{eV})$, as observed in Fig. 3(a), but with activation energies around $35 \mathrm{meV}$ and $70 \mathrm{meV}$, respectively. These values are far from being associated to thermal escape of carriers out to the barriers, as occurs in single layer QWR samples. The most outstanding difference between single and stacked QWR's is the partial coupling between them along the vertical direction, more important for excited states. In this way, the carrier tunneling time between wires in the same stack is expected to be shorter than the exciton recombination time at every temperature. That is, the excitonic recombination can take place at either the same wire where excitons are photogenerated, or any other in the stack. However, even if we can expect an effective exciton radiative lifetime slightly different from that in single wires, this situation does not explain the thermal PL quenching unless a nonradiative channel could be opened. Thermal escape of carrier towards the barriers in multilayer samples can be inhibited due to the smaller electron and hole confinement energies in the stacked wires, because they are taller than the average wire height in single layer samples. The most simple origin for this nonradiative channel is the existence of impurities in the InP material surrounding the InAs wires, even if they are not evidenced in single layer samples. This nonradiative mechanism would agree with the greater activation energy measured for the family stack emitting at $0.728 \mathrm{eV}$, given the larger energy difference between the carrier ground state and the deep impurity level (at a certain energy from the conduction band of the InP). More work is needed to give insight into this subject.

In summary, stacked InAs/InP self-assembled QWR multilayers have been successfully grown by MBE by using InP as spacer material. We have found that spacer layer thickness fully determines the structural and optical properties of the multilayer structure, being observed a strong improvement of the homogeneity and uniformity of the QWR stacks, in the case of an appropriate layer spacing. For a 5-nm-thick spacer layer, most of the wires are stacked and two wire stack families are identified by PL measurements. The wire height fluctuation in each family is around $2 \mathrm{ML}$, in agreement with the structural characterization. The exciton recombination dynamics in the WSL cannot be associated to thermal escape of carriers, as occur in single layer QWR samples, but more probably to carrier trapping in deep levels.

This work has been supported by the spanish CICYT under the Project no. TIC99-1035-C02 and European Commission GROWTH program NANOMAT project, Contract no. G5RD-CT-2001-00545. Authors from University of Cádiz also acknowledge support from the Junta de Andalucía under the group TEP-0120. TEM measuments were carried out in DME, SCCYT, University of Cádiz.

*Email address: martinep@uv. es

${ }^{1}$ Q. Xie, A. Madhukar, P. Chen, and N.P. Kobayashi, Phys. Rev. Lett. 75, 2542 (1995).

${ }^{2}$ J. Tersoff, C. Teichert, and M.G. Lagally, Phys. Rev. Lett. 76, 1675 (1996).

${ }^{3}$ R. Heitz, A. Kalburge, Q. Xie, M. Grundmann, P. Chen, A. Hoffmann, A. Madhukar, and D. Bimberg, Phys. Rev. B 57, 9050 (1998).

${ }^{4}$ P. Frigeri, A. Bosacchi, S. Franchi, P. Allegri, and V. Avanzini, J. Cryst. Growth 201/202, 1136 (1999).

${ }^{5}$ E. Petitprez, N.T. Moshegov, J.E. Marega, A. Mazel, D. Dorignac, and R. Fourmeaux, J. Vac. Sci. Technol. B 18, 1493 (2000).

${ }^{6}$ H. Li, J. Wu, Z. Wang, and T. Daniels-Race, Appl. Phys. Lett. 75, 1173 (1999).

${ }^{7}$ C. Walther, W. Hoerstel, H. Niehus, J. Erxmeyer, and W.T. Mas-

selink, J. Cryst. Growth 209, 572 (2000).

${ }^{8}$ J. Braoult, M. Gendry, O. Marty, M. Pitaval, J. Olivares, G. Grenet, and G. Hollinger, Appl. Surf. Sci. 162-163, 584 (2000).

${ }^{9}$ J. Wu, Y.P. Zeng, Z.Z. Sun, F. Lin, B. Xu, and Z.G. Zeng, J. Cryst. Growth 219, 180 (2000).

${ }^{10}$ H.R. Gutiérrez, M.A. Cotta, and M.M.G. de Carvalho, Appl. Phys. Lett. 79, 3854 (2001).

${ }^{11}$ L. González, J.M. García, R. García, J. Martínez-Pastor, C. Ballesteros, and F. Briones, Appl. Phys. Lett. 76, 1104 (2000).

${ }^{12}$ B. Alén, J. Martínez-Pastor, A. García-Cristóbal, L. González, and J.M. García, Appl. Phys. Lett. 78, 4025 (2001).

${ }^{13}$ J.M. García, L. González, M.U. González, J.P. Silveira, Y. González, and F. Briones, J. Cryst. Growth 227/228, 975 (2001).

${ }^{14}$ B. Szafran, S. Bednarek, and J. Adamowski, Phys. Rev. B 64, 125301 (2001). 